Article

\title{
Baeyer-Villiger-Including Domino Two-Step Oxidations of $\beta$-O-Substituted Primary Alcohols: Reflection of the Migratory Aptitudes of O-Substituted Alkyl Group in the Outcome of the Reaction
}

\author{
Tom Targel and Moshe Portnoy * (B) \\ School of Chemistry, Raymond and Beverly Sackler Faculty of Exact Sciences, Tel Aviv University, \\ Tel Aviv 6997801, Israel; tomtargel@mail.tau.ac.il \\ * Correspondence: portnoy@tauex.tau.ac.il
}

Received: 15 October 2020; Accepted: 30 October 2020; Published: 3 November 2020

check for updates

\begin{abstract}
Following the recent development of the one-pot two-step oxidation protocol for primary $\beta$-alkoxy alcohols, additional primary $\beta$-oxy alcohols were examined under similar conditions. The outcome of these reactions closely follows the migratory aptitudes of the related $\alpha$-oxy-alkyls in Baeyer-Villiger oxidation vs. that of hydrogen. Thus, these experiments helped to establish the correct order of these aptitudes for $\alpha$-oxy-alkyls. Furthermore, in the case of primary $\beta$, $\beta$-dialkoxyalcohols, the formation of dialkoxymethyl formates by the domino oxidation reaction was followed by secondary reactions, forming a number of interesting products.
\end{abstract}

Keywords: oxidations; organocatalysis; domino reactions; synthetic methods; migratory aptitudes

\section{Introduction}

Oxidation of secondary alcohols usually leads to ketones as end products [1], and only the Baeyer-Villiger (BV) reaction [2-5], inserting oxygen between the carbonyl and an adjacent carbon and, thus, forming an ester with an interrupted carbon skeleton, could lead to an additional oxidation completing a two-step oxidation sequence [6-19]. In the case of the primary alcohols, two-stage oxidation is much more common and leads predominantly to carboxylic acids [20], which are formed from aldehydes via the Baeyer-Villiger pathway or one of the alternative mechanisms at the second step. Only rarely, in the case of electron-rich benzaldehydes or aldehydes with a nitrogen- or an oxygen-substituted $\alpha$-carbon, BV oxidation of aldehydes results in the opposite regioselectivity, leading to hemiacetal formates [21-31]. Surprisingly, prior to our work only scarce examples of two-step BV-involving oxidation of primary alcohols to esters in two successive, but separate steps, were reported [28-31], while such one-pot oxidations have never been explored. Recently, we reported an unprecedented and efficient one-pot procedure for transformation of primary $\beta$-alkoxy alcohols into the corresponding hemiacetal formates [32]. Similarly, secondary $\beta$-alkoxy alcohols can be converted in a one-pot fashion into hemiacetal esters [33]. This process, combining TEMPO-based catalytic and $m$ CPBA-induced stoichiometric steps (Scheme 1a, TEMPO = (2,2,6,6-tetramethylpiperidin-1-yl)oxyl, $m \mathrm{CPBA}=m$-chloroperbenzoic acid), reflects the stronger migratory aptitude of alkoxyalkyls compared to that of hydrogen or simple alkyl in the BV step. 


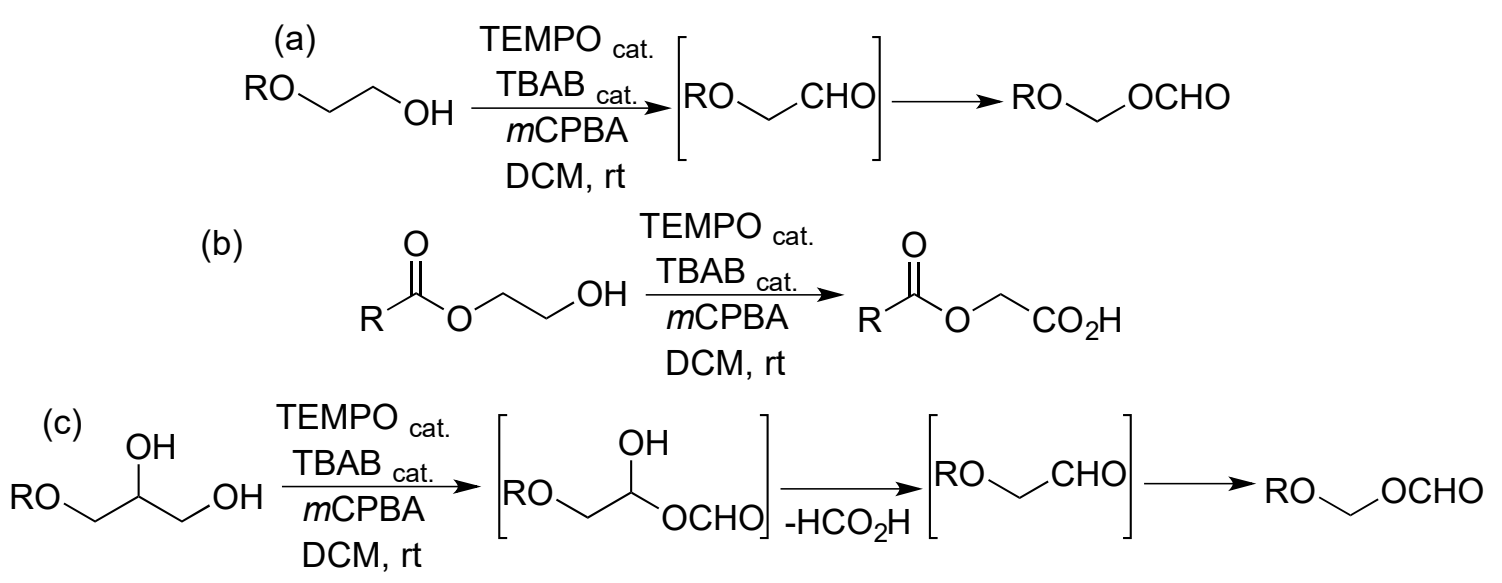

Scheme 1. Previously published reactions of $\beta$-O-substituted primary alcohols under one-pot oxidation conditions: (a) $\beta$-alkoxy alcohols; (b) $\beta$-acyloxy alcohols; (c) $\gamma$-alkoxy- $\beta$-hydroxyalcohols [32].

While attempting to expand the new methodology to additional types of substrates we found that the regioselectivity of the BV step is strongly dependent on the nature of the oxy-substituent on the $\alpha$-to-carbonyl carbon. Thus, $\beta$-acyloxy alcohols yielded carboxylic acids, while $\gamma$-alkoxy- $\beta$-hydroxyalcohols formed transient gem-diol monoesters, which underwent an additional oxidation step after eliminating formic acid (Scheme 1b,c). Obviously, the course of the domino reaction and, particularly, of the second (Baeyer-Villiger) step reflects the migratory aptitudes of various $\alpha$-O-substituted alkyl groups. It occurred to us that the reaction enables easy comparison of these aptitudes with that of hydrogen as the reference point [34] (Scheme 2).

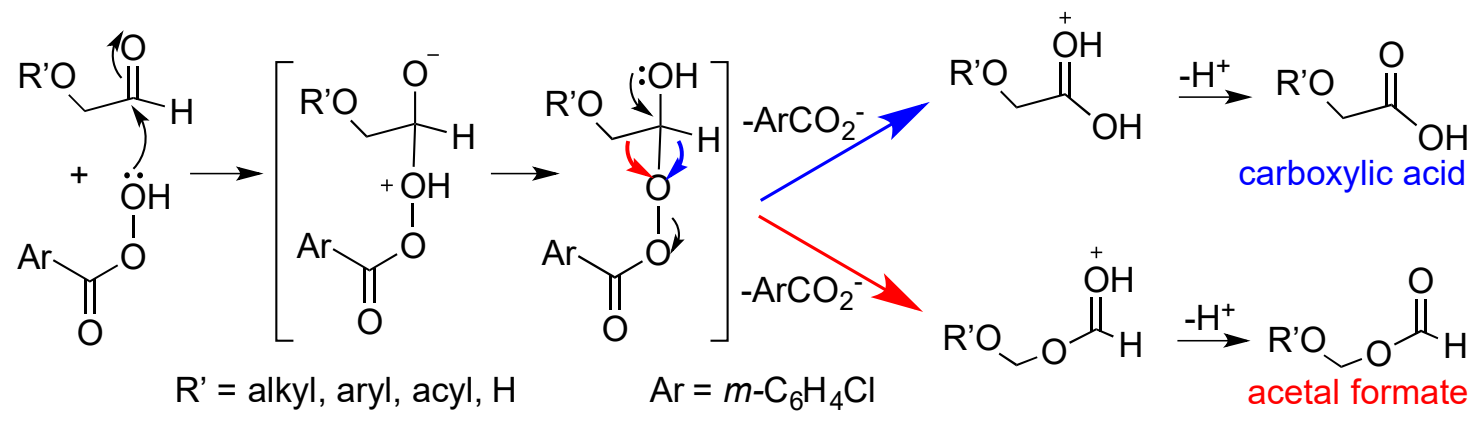

Scheme 2. The migrating group preference in the Criegee intermediate [2-5] defines the outcome of the reaction: hemiacetal formates (red arrows) vs. carboxylic acids (blue arrows).

With this notion in mind, we turned to study the reaction of other types of $\beta$-O-substituted primary alcohols under the abovementioned one-pot reaction conditions, aiming both to expand the scope of the reaction and compare the migratory aptitudes of additional oxyalkyl groups in BV oxidation. Herein we describe the results of these studies.

\section{Results and Discussion}

Our previous experiments already demonstrated the following order of migratory aptitudes: $\alpha$-acyloxyalkyl $<\mathrm{H}<\alpha$-alkoxyalkyl. The position of $\alpha$-hydroxyalkyl should be to the right of $\mathrm{H}$ in this series, in line with the abovementioned formation of transient gem-diol monoesters from $\beta$-hydroxy alcohols. Furthermore, the course of the reaction of 1,4-anhydroerythritol (a secondary alcohol, Scheme 3) hints that the migratory aptitude of $\alpha$-hydroxyalkyl is stronger than that of $\alpha$-alkoxyalkyl $[32,35]$. 


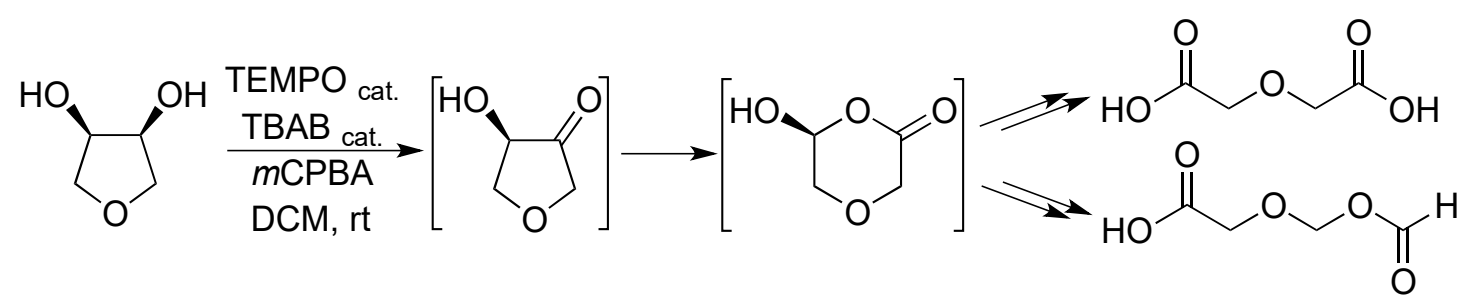

Scheme 3. Preference for the migration of the hydroxy alkyl over alkoxyalkyl in the domino reaction [32].

The position of some other oxyalkyl groups in the series describing their migratory aptitudes remained, however, unclear. Accordingly, we tested a number of additional primary alcohol substrates in the domino oxidation reaction (Table 1). 2-phenoxyethanol (1a), for instance, exhibited slow conversion into both the corresponding hemiacetal formate $\mathbf{2 a}$ and the corresponding carboxylic acid 3a [36] (entry 1). In order to achieve full consumption of the starting material, the TEMPO/TBAB loading should be increased to 5 molar \%, while that of $m$ CPBA to 6.6 equiv. Under these conditions, a ratio of 1:0.4 between the hemiacetal formate product and the carboxylic acid product is achieved. This ratio, as well as the reaction rate, indicate that though the propensity of phenoxymethyl to migrate during the $\mathrm{BV}$ reaction is stronger than that of hydrogen, it is so only by a relatively small margin.

Table 1. Reactions of additional substrates under domino oxidation conditions. ${ }^{1}$

\begin{tabular}{|c|c|c|c|c|}
\hline Entry & Substrate & Time (h) & Consumption (\%) & Products (Product Ratio) \\
\hline 1 & & 3 & 100 & (1: 0.4$)$ \\
\hline 2 & & 17 & 82 & \\
\hline 3 & & 3 & 100 & \\
\hline 4 & & 3 & 87 & \\
\hline 5 & & 1 & 100 & \\
\hline 6 & & 1 & 100 & \\
\hline 7 & & 1 & 100 & $5 \mathrm{~g}$ (maior) \\
\hline 8 & & 4 & 100 & \\
\hline
\end{tabular}

${ }^{1}$ Reaction conditions: 1 equiv of substrate with 2.2 to 6.6 equiv of $m \mathrm{CPBA}, 2$ to 5 molar $\%$ TEMPO, 2 to 5 molar $\%$ tetra- $n$-butylammonium bromide (TBAB), dichloromethane (DCM) $(8 \mathrm{~mL} / \mathrm{mmol}$ substrate), rt. For exact amounts for each entry, see the Materials and Methods Section. 
Subsequently, we decided to examine primary alcohols substituted at the $\beta$-position with a single acetal oxygen (i.e., by alkoxyalkoxy group, entries $2-4$ ). When substrates $\mathbf{1 b}$ and $\mathbf{1 c}$ were subjected to the reaction conditions [37], the corresponding carboxylic acids $3 \mathbf{b}$ and $3 \mathbf{c}$ were invariably formed as sole products. In the case of similarly substituted $\mathbf{1 d}$, a mixture of products, with carboxylic acid $\mathbf{3 d}$ being the major product, was produced. Four additional minor products are two diastereomeric pairs of hemiacetal esters, formates and $m$-chlorobenzoates, presumably derived from a single hemiacetal formate product $\mathbf{2} \mathbf{d}$ (Scheme 4). The overall ratio between $\mathbf{3} \mathbf{d}$ and $\mathbf{2} \mathbf{d}$-derived products was 6.7:1. The results of the experiments with alcohols $\mathbf{1} \mathbf{b}-\mathbf{d}$ demonstrate that the migratory aptitude of alkyls substituted by a single acetal oxygen is lower than that of hydrogen, but probably higher than that of acyloxyalkyls. Furthermore, the substrates of this kind cannot be successfully converted into hemiacetal esters under our domino reaction conditions.

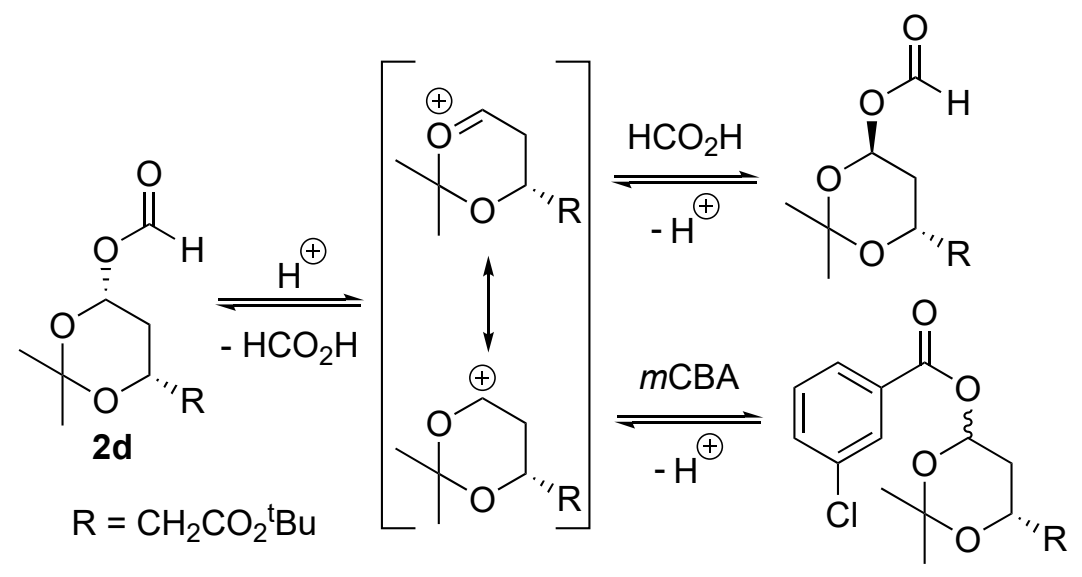

Scheme 4. Secondary transformation of the hemiacetal formate product $\mathbf{2 d}$.

The next type of substrates we wanted to examine were primary alcohols with an acetal carbon as the $\beta$-carbon (i.e., with the $\beta$-carbon substituted by two alkoxy groups). Three compounds of this type, 1e-g, were prepared (Scheme 5) and examined under standard domino reaction conditions (Table 1, Entries 5-7). If such a substrate undergoes the reaction in a mode similar to that of $\beta$-alkoxy alcohols, one may expect generation of 2-formyloxy-1,3-dioxolane or -dioxane products $\mathbf{2 e - g}$ (Scheme 6). While rare, such dialkoxymethyl carboxylates are not unprecedented [38]. However, such formates are particularly unstable and, accordingly, under the reaction conditions, their subsequent hydrolytic decomposition is possible. Indeed, diol mono-formate esters $4 \mathbf{e}, \mathbf{f}$ were identified as the major products of the reaction of substrates $\mathbf{1 e}, \mathbf{f}$, presumably via 2-step hydrolysis of $\mathbf{2 e}, \mathbf{f}$ (Scheme 6). In the reaction mixture of $\mathbf{1 e}$, minor products $\mathbf{5 e - 1}$ and $\mathbf{5 e - 2}$, presumably resulting from interception of one of the hydrolysis intermediates by the substrate or the major product, were observed. In the case of substrate $1 \mathrm{~g}$, diol mono-formate $4 \mathrm{~g}$ constitutes the minor product of the reaction, while the major product was identified as the cyclic carbonate $\mathbf{5 g}$, likely formed by an additional TEMPO-catalyzed oxidation of one of the hydrolytic intermediates (Scheme 6) [39]. These results prove that $\beta, \beta$-dialkoxy alcohols react in the domino oxidation reaction in a way similar to $\beta$-alkoxy alcohols, but form unstable products. Furthermore, it demonstrated that the migratory aptitude of the $\alpha$, $\alpha$-dialkoxymethyl in the BV reaction is higher than that of hydrogen. Finally, these experiments demonstrated selective formation of diol monoesters from diols by their conversion to hydroxyacetaldehyde-derived acetals, followed by the domino oxidation-hydrolysis sequence.

In order to compare the aptitudes of $\alpha, \alpha$-dialkoxyalkyl and $\alpha$-alkoxyalkyl, we examined the reaction of a secondary alcohol $\mathbf{1 h}$ with both groups flanking the hydroxyl-bearing carbon (Table 1, Entry 8 ). In this experiment the formation of product $\mathbf{2 h}$, generated by oxidation of the secondary alcohol followed by the oxygen insertion between the formed carbonyl and the alkoxy-substituted neighboring carbon, indicates that the migratory tendency of $\alpha$-alkoxyalkyl is stronger (in the BV reaction). 


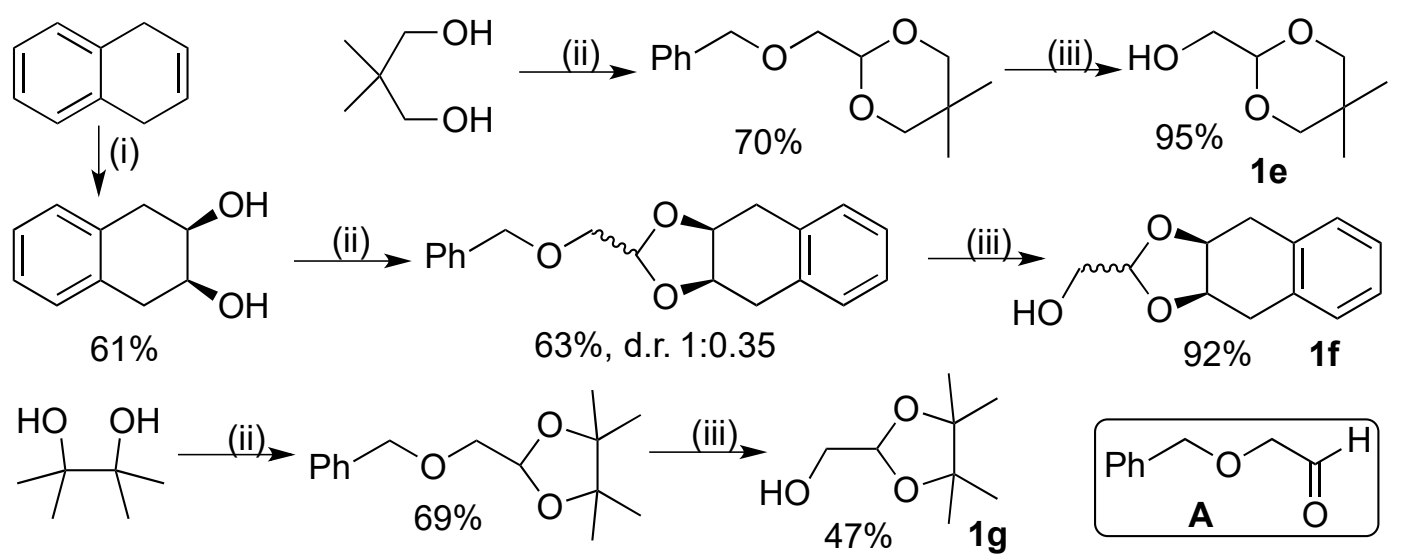

Scheme 5. Synthesis of compounds 1e-g. Reagents and conditions: (i) NMO (N-methylmorpholine $\mathrm{N}$-oxide), $\mathrm{OsO}_{4}$ cat. ${ }^{\mathrm{t}} \mathrm{BuOH} /$ acetone/ $\mathrm{H}_{2} \mathrm{O}$, rt; (ii) $\mathbf{A}$, $\mathrm{TsOH}_{\text {cat. }}$, toluene, reflux; (iii) $\mathrm{H}_{2}, \mathrm{Pd} / \mathrm{C}_{\text {cat., }}, \mathrm{MeOH}$, rt.

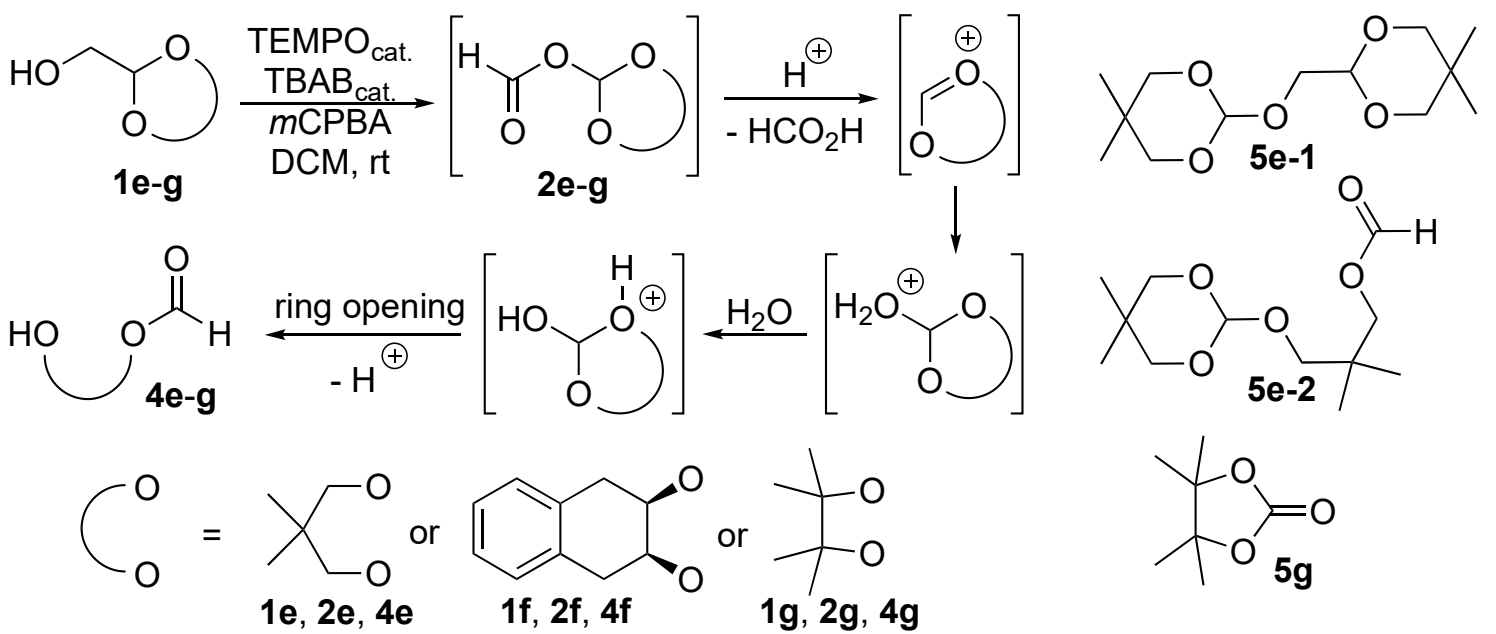

Scheme 6. Domino oxidation of substrates $(\mathbf{1}-\mathbf{g})$ and secondary reactions of the formed dialkoxymethyl carboxylates.

Another aspect that was examined during our experiments is the influence of the solvent on the domino reaction course. We focused our attention on two substrates, which in DCM gave both the hemiacetal formate and the carboxylic acid products, namely $\mathrm{Bz}\left(\mathrm{OCH}_{2} \mathrm{CH}_{2}\right)_{2} \mathrm{OH}$ (1i) that was reported in our previous communication and $\mathbf{1 d}$ (Table 2). Three solvents, apolar benzene, moderately polar ethyl acetate and polar acetonitrile, were compared to the originally used DCM. In both cases the best solvent for the formation of hemiacetal esters was benzene, while acetonitrile, the most polar of the three solvents, most favored the formation of carboxylic acid. Lower conversions observed in ethyl acetate are attributed to the absence of TBAB in these experiments due to its insolubility in this solvent. In the past, similar solvent dependence was observed in oxidation of aldehyde by dioxygen or peracids, with the increase in acid formation attributed to the disruption of the transition state, preferentially leading to acetal formates, by polar solvents [40]. Interestingly, the opposite trend was observed in aqueous organic solvents in the related Dakin reaction [41]. 
Table 2. Influence of the solvent on the distribution of the reaction products ${ }^{1}$.

\begin{tabular}{ccccc}
\hline Entry & Substrate & Solvent & Consumption (\%) & Product Ratio $^{\mathbf{2}}$ \\
\hline 1 & $\mathbf{1 i}$ & benzene & 100 & $14: 1$ \\
2 & $\mathbf{1 i}$ & DCM & 100 & $11: 1$ \\
$3^{3}$ & $\mathbf{1 i}$ & EtOAc & 47 & $10: 1$ \\
4 & $\mathbf{1 i}$ & ACN & 96 & $3.1: 1$ \\
5 & $\mathbf{1 d}$ & benzene & 86 & $1: 4.9$ \\
6 & $\mathbf{1 d}$ & DCM & 87 & $1: 6.7$ \\
$7^{3}$ & $\mathbf{1 d}$ & EtOAc & 79 & $1: 24$ \\
8 & $\mathbf{1 d}$ & ACN & 100 & Acid only \\
\hline
\end{tabular}

${ }^{1}$ Reaction conditions: 1 equiv of substrate with 2.2 equiv of $m$ CPBA, 2 or 5 molar $\%$ TEMPO, 2 or 5 molar $\%$ TBAB, solvent ( $8 \mathrm{~mL} / \mathrm{mmol}$ substrate), $1-3 \mathrm{~h}$, rt. For exact amounts and time for each entry, see Materials and Methods Section.

${ }^{2}$ Ratio between the hemiacetal ester(s) and the carboxylic acid products. ${ }^{3}$ Without TBAB.

\section{Materials and Methods}

\subsection{Materials and Techniques}

All reactions, requiring anhydrous conditions, were conducted under an atmosphere of nitrogen in oven-dried glassware in dry solvents. Commercially available materials were purchased at the highest available purity and were used as received. Thin layer chromatography (TLC) was performed on silica gel plates Merck $60 \mathrm{~F}_{254}$, and the compounds were visualized by irradiation with UV light or by staining with $\mathrm{KMnO}_{4}$. Flash column chromatography was carried out using silica gel Merck 60 (particle size $0.040-0.063 \mathrm{~mm})$; the eluent is given in parentheses. ${ }^{1} \mathrm{H}$ NMR $(400 \mathrm{MHz})$ and ${ }^{13} \mathrm{C} \mathrm{NMR}(100 \mathrm{MHz})$ spectra were recorded on Bruker AVANCE-400 spectrometers, in DMSO- $d_{6}$ with residual DMSO- $d_{5}$ $\left({ }^{1} \mathrm{H}, 2.50 \mathrm{ppm}\right)$ or DMSO- $d_{6}\left({ }^{13} \mathrm{C}, 39.52 \mathrm{ppm}\right)$ as an internal shift calibration standard; or in $\mathrm{CDCl}_{3}$ with residual $\mathrm{CHCl}_{3}\left({ }^{1} \mathrm{H}, 7.26 \mathrm{ppm}\right)$ or $\mathrm{CDCl}_{3}\left({ }^{13} \mathrm{C}, 77.16 \mathrm{ppm}\right)$ as an internal shift calibration standard. MS analyses were conducted on Waters SYNAPT instrument. (See the Supplementary Materials) IR spectra were recorded on Bruker Tensor 27 with Platinum ATR.

\subsection{Synthesis of Substrates}

\subsubsection{Synthesis of [(2S,4S)-2-Phenyl-1,3-Dioxan-4-yl]Methanol (1b):}

A solution of (S)-1,2,4-butanetriol (531 mg, $5.0 \mathrm{mmol}, 1.0$ equiv), benzaldehyde dimethyl acetal ( $841 \mathrm{mg}$, $5.5 \mathrm{mmol}$, 1.1 equiv) and camphorsulfonic acid ( $59 \mathrm{mg}, 0.25 \mathrm{mmol}, 5 \mathrm{~mol} \%$ ) in $\mathrm{CH}_{2} \mathrm{Cl}_{2}(21 \mathrm{~mL})$ was stirred at room temperature for $20 \mathrm{~h}$. Imidazole was added in one portion $(34 \mathrm{mg}, 0.5 \mathrm{mmol}$, 0.1 equiv) and the resulting mixture was concentrated in vacuo. The residue was purified by flash chromatography (silica gel, 1:3 to 3:1 EtOAc/hexane) to yield the product as a colorless oil (399 mg, 41\%) [42].

TLC: $\mathrm{R}_{\mathrm{f}}=0.4$ (3:1 EtOAc/hexane eluent). ${ }^{1} \mathrm{H}-\mathrm{NMR}\left(400 \mathrm{MHz}, \mathrm{CDCl}_{3}\right) \delta 7.51-7.48(\mathrm{dd}, J=7.6$, $1.8 \mathrm{~Hz}, 2 \mathrm{H}), 7.40-7.33(\mathrm{~m}, 3 \mathrm{H}), 5.55(\mathrm{~s}, 1 \mathrm{H}), 4.30(\mathrm{ddd}, J=11.4,5.1,1.2 \mathrm{~Hz}, 1 \mathrm{H}), 4.04-3.98(\mathrm{~m}, 1 \mathrm{H})$, superimposed by 4.02-3.95 (td, $J=11.9,2.4 \mathrm{~Hz}, 1 \mathrm{H}), 3.73-3.62(\mathrm{~m}, 2 \mathrm{H}), 2.16(\mathrm{t}, J=6.3 \mathrm{~Hz}, 1 \mathrm{H}), 1.98-1.87$ $(\mathrm{qd}, J=12.4,5.2 \mathrm{~Hz}, 1 \mathrm{H}), 1.47-1.43(\mathrm{dtd}, J=13.2,2.5 \mathrm{~Hz}, 1.4 \mathrm{~Hz}, 1 \mathrm{H}) .{ }^{13} \mathrm{C}-\mathrm{NMR}\left(100 \mathrm{MHz}, \mathrm{CDCl}_{3}\right)$ : $\delta 138.5,129.1,128.4,126.2,101.4,77.7,66.7,65.8,26.9$.

\subsubsection{Synthesis of (5,5-Dimethyl-1,3-Dioxan-2-yl)Methanol (1e):}

2-((benzyloxy)methyl)-5,5-dimethyl-1,3-dioxane [43]: In a round-bottom flask equipped with a Dean-Stark apparatus, a water-cooled condenser and a magnetic stir bar, benzyloxyacetaldehyde (760 mg, 1.0 equiv, $5.06 \mathrm{mmol}$ ), 2.2-dimethyl-1,3-propanediol (633 mg, 1.2 equiv, $6.07 \mathrm{mmol}$ ) and p-toluenesulfonic acid monohydrate $(43 \mathrm{mg}, 0.23 \mathrm{mmol}, 4.5 \mathrm{~mol} \%)$ were dissolved in toluene $(2 \mathrm{~mL})$, refluxed for $4 \mathrm{~h}$, and were subsequently cooled to room temperature. Triethylamine $(0.6 \mathrm{~mL}, 0.85$ equiv, $4.3 \mathrm{mmol}$ ) was added and the solvent was removed under reduced pressure. The residue was purified 
using column chromatography (silica gel, 1:9 EtOAc/hexane) to give the desired product (835 mg, $3.53 \mathrm{mmol}, 70 \%$ yield) as a colorless oil.

TLC: $R_{\mathrm{f}}=0.3\left(1: 9 \mathrm{EtOAc} /\right.$ hexane eluent). ${ }^{1} \mathrm{H}-\mathrm{NMR}\left(400 \mathrm{MHz}, \mathrm{CDCl}_{3}\right): \delta 7.31(\mathrm{~m}, 5 \mathrm{H})$, $4.66(\mathrm{t}, J=4.6 \mathrm{~Hz}, 1 \mathrm{H}), 4.60(\mathrm{~s}, 2 \mathrm{H}), 3.64(\mathrm{~d}, J=11.2 \mathrm{~Hz}, 2 \mathrm{H}), 3.55(\mathrm{~d}, J=4.6 \mathrm{~Hz}, 2 \mathrm{H}), 3.45(\mathrm{~d}, J=10.7 \mathrm{~Hz}$, 2H), $1.20(\mathrm{~s}, 3 \mathrm{H}), 0.73(\mathrm{~s}, 3 \mathrm{H}) .{ }^{13} \mathrm{C}-\mathrm{NMR}\left(100 \mathrm{MHz}, \mathrm{CDCl}_{3}\right): \delta 137.9,128.5,128.1,127.9,100.2,77.20,73.8$, $71.4,30.5,23.1,22.0$.

(5,5-dimethyl-1,3-dioxan-2-yl)methanol (1e) [43]: 2-((benzyloxy)methyl)-5,5-dimethyl-1,3-dioxane $(800 \mathrm{mg}, 3.38 \mathrm{mmol})$ was dissolved in methanol $(5 \mathrm{~mL})$ in a round-bottom flask equipped with a magnetic stir bar. A small spoonful of $5 \% \mathrm{Pd} / \mathrm{C}$ was added, and a hydrogen-filled balloon was attached to the flask via a suitable adapter. After flushing the flask with hydrogen a few times, the mixture was allowed to stir overnight at room temperature. The mixture was then filtered through celite and the resulting clear solution was concentrated under reduced pressure to give the desired product $(470 \mathrm{mg}$, $3.21 \mathrm{mmol}, 95 \%$ yield) as a colorless oil, which was used without further purification.

${ }^{1} \mathrm{H}-\mathrm{NMR}\left(400 \mathrm{MHz}, \mathrm{CDCl}_{3}\right): \delta 4.54(\mathrm{t}, J=4.4 \mathrm{~Hz}, 1 \mathrm{H}), 3.64(\mathrm{~m}, 4 \mathrm{H}), 3.47(\mathrm{~d}, J=10.6 \mathrm{~Hz}, 2 \mathrm{H})$, $1.93(\mathrm{t}, J=6.6 \mathrm{~Hz}, 1 \mathrm{H}), 1.19(\mathrm{~s}, 3 \mathrm{H}), 0.74(\mathrm{~s}, 3 \mathrm{H}) .{ }^{13} \mathrm{C}-\mathrm{NMR}\left(100 \mathrm{MHz}, \mathrm{CDCl}_{3}\right): \delta 100.5,77.1,64.1,30.6$, 23.0, 21.9.

\subsubsection{Synthesis of ((3ar,9as)-3a,4,9,9a-Tetrahydronaphtho[2,3-d][1,3]dioxol-2-yl)methanol (1f)}

(2R,3S)-1,2,3,4-tetrahydronaphthalene-2,3-diol [44]: 1,4-dihydronapthalene (326 mg, $2.50 \mathrm{mmol}$, 1.0 equiv), $\mathrm{NMO}\left(590 \mathrm{mg}, 5.04 \mathrm{mmol}, 2\right.$ equiv) and $\mathrm{OsO}_{4}(4 \% \mathrm{w} / \mathrm{w}$ in water, $0.12 \mathrm{~mL}, 0.02 \mathrm{mmol}$, $0.8 \mathrm{~mol} \%$ ) were dissolved in a mixture of $3.5 \mathrm{~mL}$ acetone, $3.5 \mathrm{~mL}$ of water and $0.35 \mathrm{~mL}$ of ${ }^{\mathrm{t}} \mathrm{BuOH}$ in a vial charged with a magnetic stir bar. The mixture was allowed to stir and was monitored by TLC every half an hour until no more starting material was observed, after an hour and a half. The solvent mixture was removed under reduced pressure, and the crude was purified by column chromatography (silica gel, 1:3 EtOAc/hexane to EtOAc) to give the desired product (253 mg, $1.53 \mathrm{mmol}, 61 \%$ yield) as a colorless liquid.

TLC: $\mathrm{R}_{\mathrm{f}}=0.3$ (9:1 EtOAc/hexane eluent). ${ }^{1} \mathrm{H}-\mathrm{NMR}\left(400 \mathrm{MHz}, \mathrm{CDCl}_{3}\right): \delta 7.11(\mathrm{~m}, 4 \mathrm{H})$, $4.11(\mathrm{t}, J=5.2 \mathrm{~Hz}, 2 \mathrm{H}), 3.01(\mathrm{~m}, 4 \mathrm{H}), 2.45(\mathrm{~s}, 2 \mathrm{H}) .{ }^{13} \mathrm{C}-\mathrm{NMR}\left(100 \mathrm{MHz}, \mathrm{CDCl}_{3}\right): \delta 133.0,129.3$, 126.4, 69.4, 34.5.

(3aR,9aS)-2-((Benzyloxy)methyl)-3a,4,9,9a-Tetrahydronaphtho[2,3-d][1,3]: The abovementioned diol (202 mg, $1.23 \mathrm{mmol}, 1.0$ equiv) and 2-(benzyloxy)acetaldehyde (181 mg, $1.21 \mathrm{mmol}, 0.98$ equiv) were dissolved in toluene $(2 \mathrm{~mL})$ in a vial charged with a magnetic stir bar, connected to a Dean-Stark apparatus. TsOH (11.4 mg, $0.06 \mathrm{mmol}, 5 \mathrm{~mol} \%)$ was added and the mixture was allowed to stir for $3.5 \mathrm{~h}$. TEA $(0.15 \mathrm{~mL})$ was added, and the mixture was concentrated under reduced pressure. The residue was purified by column chromatography (silica gel, 1:9 to 1:4 EtOAc/hexane) to give a 1:0.35 diastereomeric mixture of the desired product ( $231 \mathrm{mg}, 0.78 \mathrm{mmol}, 63 \%$ yield) as a yellow oil.

TLC: $\mathrm{R}_{\mathrm{f}}=0.3$ (1:4 EtOAc/hexane eluent).

Major diastereoisomer: ${ }^{1} \mathrm{H}-\mathrm{NMR}\left(400 \mathrm{MHz}, \mathrm{CDCl}_{3}\right): \delta 7.23-7.34(\mathrm{~m}, 5 \mathrm{H}), 7.14-7.20(\mathrm{~m}, 4 \mathrm{H})$,

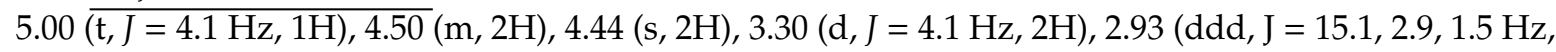
2H), 2.81 (ddd, J = 15.0, 3.0, $1.6 \mathrm{~Hz}, 2 \mathrm{H}) .{ }^{13} \mathrm{C}-\mathrm{NMR}\left(100 \mathrm{MHz}, \mathrm{CDCl}_{3}\right): \delta 138.0,135.1,128.4(2 \mathrm{C}), 127.9$, $127.7,126.8,101.8,75.2,73.6,70.6,33.9$.

Minor diastereoisomer: ${ }^{1} \mathrm{H}-\mathrm{NMR}\left(400 \mathrm{MHz}, \mathrm{CDCl}_{3}\right): \delta 7.23-7.34(\mathrm{~m}, 5 \mathrm{H}), 7.14-7.20(\mathrm{~m}, 4 \mathrm{H})$, $4.95(\mathrm{t}, J=3.9 \mathrm{~Hz}, 1 \mathrm{H}), 4.68(\mathrm{~m}, 2 \mathrm{H}), 4.56(\mathrm{~s}, 2 \mathrm{H}), 3.43(\mathrm{~d}, J=3.9 \mathrm{~Hz}, 2 \mathrm{H}), 2.97(\mathrm{ddd}, \mathrm{J}=14.9,3.0,1.4 \mathrm{~Hz}$, 2H), $2.83(\mathrm{ddd}, \mathrm{J}=15.0,3.0,1.6 \mathrm{~Hz}, 2 \mathrm{H}) .{ }^{13} \mathrm{C}-\mathrm{NMR}\left(100 \mathrm{MHz}, \mathrm{CDCl}_{3}\right): \delta 138.0,135.3,128.5,128.3,127.9$, $127.8,127.0,102.7,75.3,73.8,71.3,34.2$.

IR (neat) $v 2912,1456,1110,751 \mathrm{~cm}^{-1}$. HRMS $\left(\mathrm{AP}^{+} / \mathrm{TOF}\right) \mathrm{m} / \mathrm{z}$ : calcd for $\mathrm{C}_{19} \mathrm{H}_{19} \mathrm{O}_{3}\left([\mathrm{M}-\mathrm{H}]^{+}\right)$ 295.1334; found 295.1338.

((3aR,9aS)-3a,4,9,9a-Tetrahydronaphtho[2,3-d][1,3]dioxol-2-yl)methanol (1f): The abovementioned diastereomeric mixture (216 mg, $0.73 \mathrm{mmol}, 1.0$ equiv) was dissolved in methanol (2.5 mL). A small spoon of activated $10 \% \mathrm{Pd} / \mathrm{C}$ was added to it in a round-bottom flask charged with a magnetic stir bar, 
a hydrogen balloon was attached and the mixture was allowed to stir for $24 \mathrm{~h}$ at room temperature. The resulting solution was filtered through celite and concentrated under reduced pressure to give a 1:0.4 diastereomeric mixture of $\mathbf{1 f}(138 \mathrm{mg}, 0.67 \mathrm{mmol}, 91 \%$ yield) as a yellow liquid, which was used in the next step without further purification.

Major diastereoisomer: ${ }^{1} \mathrm{H}-\mathrm{NMR}\left(400 \mathrm{MHz}, \mathrm{CDCl}_{3}\right): \delta 7.20(\mathrm{~m}, 4 \mathrm{H}), 4.91(\mathrm{t}, J=2.4 \mathrm{~Hz}, 1 \mathrm{H})$, $4.60(\mathrm{~m}, 2 \mathrm{H}), 3.46(\mathrm{~s}, 2 \mathrm{H}), 3.00(\mathrm{~m}, 2 \mathrm{H}), 2.76(\mathrm{~m}, 2 \mathrm{H}) .{ }^{13} \mathrm{C}-\mathrm{NMR}\left(100 \mathrm{MHz}, \mathrm{CDCl}_{3}\right): \delta 134.8,128.5,127.1$, $101.7,75.2,62.0,33.7$.

Minor diastereoisomer: ${ }^{1} \mathrm{H}-\mathrm{NMR}\left(400 \mathrm{MHz}, \mathrm{CDCl}_{3}\right): \delta 7.20(\mathrm{~m}, 4 \mathrm{H}), 4.76(\mathrm{t}, J=3.4 \mathrm{~Hz}, 1 \mathrm{H})$, $4.71(\mathrm{~m}, 2 \mathrm{H}), 3.51(\mathrm{~d}, J=3.0 \mathrm{~Hz}, 2 \mathrm{H}), 3.00(\mathrm{~m}, 2 \mathrm{H}), 2.83(\mathrm{~m}, 2 \mathrm{H}) .{ }^{13} \mathrm{C}-\mathrm{NMR}\left(100 \mathrm{MHz}, \mathrm{CDCl}_{3}\right): \delta 135.2$, $128.3,127.1,103.3,75.5,63.1,34.3$.

IR (neat) $v 3422,2916,1458,1137,1049,750 \mathrm{~cm}^{-1}$. HRMS (ES+TOF) m/z: calcd for $\mathrm{C}_{12} \mathrm{H}_{14} \mathrm{O}_{3} \mathrm{Na}$ $\left([\mathrm{M}+\mathrm{Na}]^{+}\right)$229.0841; found 229.0835.

\subsubsection{Synthesis of (4,4,5,5-Tetramethyl-1,3-dioxolan-2-yl)methanol (1g):}

2-((benzyloxy)methyl)-4,4,5,5-tetramethyl-1,3-dioxolane: Pinacol (718 mg, $6.07 \mathrm{mmol}, 1.2$ equiv) and 2-(benzyloxy)acetaldehyde (760 mg, $5.06 \mathrm{mmol}, 1.0$ equiv) were dissolved in toluene $(10 \mathrm{~mL})$ in a round-bottom flask, equipped with a Dean-Stark apparatus and charged with a magnetic stir bar. $\mathrm{TsOH}$ (48 mg, $0.25 \mathrm{mmol}, 5 \mathrm{~mol} \%$ ) was added and the mixture was allowed to stir overnight. TEA $(0.50 \mathrm{~mL})$ was added, and the mixture was concentrated under reduced pressure. The residue was purified by column chromatography (silica gel, 1:19 to 1:4 EtOAc/hexane) to give the desired product (870 mg, $3.48 \mathrm{mmol}, 68.8 \%$ yield) as a yellow oil.

TLC: $\mathrm{R}_{\mathrm{f}}=0.35$ (1:4 EtOAc/hexane eluent). ${ }^{1} \mathrm{H}-\mathrm{NMR}\left(400 \mathrm{MHz}, \mathrm{CDCl}_{3}\right): \delta$ 7.24-7.37 (m, 5H), $5.19(\mathrm{t}, J=4.5 \mathrm{~Hz}, 1 \mathrm{H}), 4.61(\mathrm{~s}, 2 \mathrm{H}), 3.49(\mathrm{~d}, J=4.5 \mathrm{~Hz}, 2 \mathrm{H}), 1.21(\mathrm{~s}, 6 \mathrm{H}), 1.20(\mathrm{~s}, 6 \mathrm{H}) .{ }^{13} \mathrm{C}-\mathrm{NMR}(100 \mathrm{MHz}$, $\mathrm{CDCl}_{3}$ ): $\delta 138.2,128.4,128.0,127.7,99.2,82.2,73.7,72.7,24.0,22.1$. IR (neat) $\vee 2978,2865,1454$, $1367,1156,1105,733,697 \mathrm{~cm}^{-1}$. HRMS (ES $/$ TOF) m/z: calcd for $\mathrm{C}_{15} \mathrm{H}_{21} \mathrm{O}_{3}\left([\mathrm{M}-\mathrm{H}]^{+}\right)$249.1491; found 249.1494 .

(4,4,5,5-tetramethyl-1,3-dioxolan-2-yl)methanol (1g): The abovementioned protected alcohol ( $870 \mathrm{mg}, 3.48 \mathrm{mmol}, 1$ equiv) was dissolved in methanol. A spoon of activated $10 \% \mathrm{Pd} / \mathrm{C}$ was added to a vial charged with a magnetic stir bar, a hydrogen balloon was attached and the mixture was allowed to stir for $24 \mathrm{~h}$ at room temperature. The resulting solution was filtered through celite and concentrated under reduced pressure to give $1 \mathrm{~g}$ ( $260 \mathrm{mg}, 1.62 \mathrm{mmol}, 47 \%$ yield) as a yellow oil, which was used in the next step without further purification.

${ }^{1} \mathrm{H}-\mathrm{NMR}\left(400 \mathrm{MHz}, \mathrm{CDCl}_{3}\right): \delta 5.09(\mathrm{t}, J=3.9 \mathrm{~Hz}, 1 \mathrm{H}), 3.60(\mathrm{dd}, J=6.5,3.9 \mathrm{~Hz}, 2 \mathrm{H})$, $1.96(\mathrm{t}, J=6.6 \mathrm{~Hz}, 1 \mathrm{H}), 1.22(\mathrm{~s}, 6 \mathrm{H}), 1.21(\mathrm{~s}, 6 \mathrm{H}) .{ }^{13} \mathrm{C}-\mathrm{NMR}\left(100 \mathrm{MHz}, \mathrm{CDCl}_{3}\right): \delta 99.6,82.5,64.9$, 24.2, 22.2. IR (neat) $v 3446,2978,2875,1447,1368,1153,1054,889 \mathrm{~cm}^{-1}$. HRMS (ES+/TOF) m/z: calcd for $\mathrm{C}_{8} \mathrm{H}_{16} \mathrm{O}_{3} \mathrm{Na}\left(\left[\mathrm{M}+\mathrm{Na}^{+}\right]^{+}\right)$183.0997; Found 183.1003.

\subsection{Oxidations and Characterization of Products}

\subsubsection{Typical Procedure for TEMPO/mCPBA Oxidation:}

The substrate (1.0 equiv, $0.1 \mathrm{mmol}$ ) was placed in a small vial charged with a magnetic stir bar, and the solutions of TEMPO $(0.1 \mathrm{M}, 20-50 \mu \mathrm{L}, 2-5$ molar $\%)$ and TBAB $(0.1 \mathrm{M}, 20-50 \mu \mathrm{L}, 2-5$ molar \%) were added by syringe at room temperature. $m \mathrm{CPBA}$ (2.2-6.6 equiv.) solution in $0.70-0.76 \mathrm{~mL} \mathrm{DCM}$ was added, resulting in a total volume of $0.80 \mathrm{~mL} \mathrm{DCM}$, and the reaction was allowed to stir for 1 to $17 \mathrm{~h}$, depending on the substrate. In some cases, the solvent was removed under reduced pressure, and the crude reaction mixture was analyzed by NMR spectroscopy in DMSO-d6. In other cases, aqueous saturated $\mathrm{NaHCO}_{3}$ solution $(5.0 \mathrm{~mL}$ ) was added, the mixture was stirred for $5 \mathrm{~min}$, the organic phase was separated, and the aqueous phase was extracted with DCM $(4 \times 2.5 \mathrm{~mL})$. Subsequently, the combined organic phase was washed with brine $(5 \mathrm{~mL})$, dried over $\mathrm{Na}_{2} \mathrm{SO}_{4}$, filtered, concentrated in vacuo, and the crude was analyzed by NMR spectroscopy. 


\subsubsection{Oxidation of 2-Phenoxyethan-2-ol (1a):}

Commercially available $1 \mathrm{a}(138 \mathrm{mg}, 1.0$ equiv, $1.0 \mathrm{mmol})$ was oxidized according to the general procedure outlined above, scaled-up by ten ( $5 \mathrm{~mol} \%$ TEMPO, $5 \mathrm{~mol} \% \mathrm{TBAB}, 3.3$ equiv. $m \mathrm{CPBA}$, $3 \mathrm{~h}$ reaction time), including the workup sequence. Purification by column chromatography (silica gel, $1-5 \%$ EtOAc/hexane) gave $2 \mathrm{a}(23 \mathrm{mg}, 0.15 \mathrm{mmol}, 15 \%$ yield) as a yellow oil.

TLC: $\mathrm{R}_{\mathrm{f}}=0.3$ (5\% EtOAc/hexane eluent). ${ }^{1} \mathrm{H}$ NMR $\left(400 \mathrm{MHz}, \mathrm{CDCl}_{3}\right): \delta 8.14(\mathrm{~s}, 1 \mathrm{H}), 7.33(\mathrm{~m}, 2 \mathrm{H})$, $7.07(\mathrm{~m}, 3 \mathrm{H}), 7.45(\mathrm{~m}, 2 \mathrm{H}), 5.86(\mathrm{~s}, 2 \mathrm{H}) .{ }^{13} \mathrm{C} \mathrm{NMR}\left(100 \mathrm{MHz}, \mathrm{CDCl}_{3}\right): \delta 159.9,156.7,129.8,123.2,116.2$, 85.4. IR (neat) v 2924, 1732, 1597, 1494, 1213, 1048, 946, 750, $691 \mathrm{~cm}^{-1}$. HRMS $\left(\mathrm{EI}^{+}\right) \mathrm{m} / \mathrm{z}$ : calcd for $\mathrm{C}_{8} \mathrm{H}_{8} \mathrm{O}_{3}\left(\mathrm{M}^{+}\right)$152.0473; found 152.0471.

\subsubsection{Oxidation of [(2S,4S)-2-Phenyl-1,3-dioxan-4-yl]methanol (1b):}

$\mathbf{1 b}$ (97 $\mathrm{mg}, 1.0$ equiv, $0.5 \mathrm{mmol}$ ) was oxidized according to the general procedure outlined above, scaled-up by five ( $2 \mathrm{~mol} \%$ TEMPO, $2 \mathrm{~mol} \% \mathrm{TBAB}, 2.2$ equiv. $m \mathrm{CPBA}$ ), excluding the workup sequence. After overnight stirring, the reaction mixture was directly loaded to a silica gel column and purified (1:1 EtOAc/hexane to EtOAc, followed by 1:9 to $1: 4 \mathrm{MeOH} / \mathrm{EtOAc})$ to give $3 \mathbf{b}$ (19 mg, $0.09 \mathrm{mmol}, 18 \%$ yield) as a colorless solid [45].

TLC: $\mathrm{R}_{\mathrm{f}}=0.25$ (1:4 MeOH/EtOAc eluent). ${ }^{1} \mathrm{H}-\mathrm{NMR}(400 \mathrm{MHz}$, methanol- $d 4) \delta$ 7.54-7.51 (m, 2H), 7.37-7.32 (m, 3H), $5.59(\mathrm{~s}, 1 \mathrm{H}), 4.47-4.44(\mathrm{dd}, J=11.4,3.0 \mathrm{~Hz}, 1 \mathrm{H}), 4.29-4.25(\mathrm{ddd}, J=11.4,4.8,1.2 \mathrm{~Hz}$, $1 \mathrm{H}), 4.08-4.02(\mathrm{td}, J=11.7,2.9 \mathrm{~Hz}, 1 \mathrm{H}), 2.02-1.90(\mathrm{~m}, 2 \mathrm{H}) .{ }^{13} \mathrm{C}-\mathrm{NMR}$ (100 MHz, methanol- $\left.d 4\right): \delta 176.0$, 139.8, 129.8, 129.0, 127.5, 102.4, 77.6, 68.1, 29.9. HRMS (ES $/$ TOF) $\mathrm{m} / \mathrm{z}$ : calcd for $\mathrm{C}_{11} \mathrm{H}_{11} \mathrm{O}_{4}\left([\mathrm{M}-\mathrm{H}]^{-}\right)$ 207.0657; found 207.0659.

3.3.4. Oxidation of (2R,3R,4S,5R,6S)-2-(hydroxymethyl)-6-methoxytetrahydro-2H-pyran-3,4,5-triyl tribenzoate $(1 \mathrm{c})$ :

Commercially available $1 \mathrm{c}(51 \mathrm{mg}, 1.0$ equiv, $0.1 \mathrm{mmol})$ was oxidized according to the general procedure outlined above ( $2 \mathrm{~mol} \%$ TEMPO, $2 \mathrm{~mol} \% \mathrm{TBAB}, 2.2$ equiv. $m \mathrm{CPBA}, 3 \mathrm{~h}$ reaction time), including the workup sequence. The product $3 \mathrm{c}$ was characterized from the crude reaction mixture without purification [46].

${ }^{1} \mathrm{H}-\mathrm{NMR}(400 \mathrm{MHz}$, DMSO-d6): $\delta 7.85(\mathrm{~d}, J=7.0,2 \mathrm{H}), 7.84(\mathrm{~d}, J=7.0 \mathrm{~Hz}, 2 \mathrm{H}), 7.77(\mathrm{~d}, J=7.4 \mathrm{~Hz}$, $2 \mathrm{H}), 7.59(\mathrm{t}, J=7.4 \mathrm{~Hz}, 1 \mathrm{H}), 7.56(\mathrm{t}, J=7.4 \mathrm{~Hz}, 1 \mathrm{H}), 7.52(\mathrm{t}, J=7.9 \mathrm{~Hz}, 1 \mathrm{H}), 7.45(\mathrm{t}, J=7.7 \mathrm{~Hz}, 2 \mathrm{H}), 7.42$ $(\mathrm{t}, J=7.7 \mathrm{~Hz}, 2 \mathrm{H}), 7.37(\mathrm{t}, J=7.7 \mathrm{~Hz}, 2 \mathrm{H}), 5.89(\mathrm{t}, J=9.7 \mathrm{~Hz}, 1 \mathrm{H}), 5.67(\mathrm{t}, J=9.7 \mathrm{~Hz}, 1 \mathrm{H}), 5.41(\mathrm{dd}$, $J=10.0,3.4 \mathrm{~Hz} 1 \mathrm{H}), 5.26(\mathrm{~d}, J=3.3 \mathrm{~Hz}, 1 \mathrm{H}), 4.17(\mathrm{~d}, J=10.0 \mathrm{~Hz}, 1 \mathrm{H}), 3.43(\mathrm{~s}, 3 \mathrm{H}) .{ }^{13} \mathrm{C}-\mathrm{NMR}(100 \mathrm{MHz}$, DMSO-d6): $\delta$ 169.1, 165.0, 164.7, 164.2, 133.1, 132.8, 132.4, 129.6, 128.80, 128.76, 128.73, 128.61, 128.55, 128.2, 128.0, 127.9, 96.2, 71.1, 70.7, 70.2, 54.8, 54.2. HRMS (ES $/$ TOF) m/z: calcd for $\mathrm{C}_{28} \mathrm{H}_{23} \mathrm{O}_{10}\left([\mathrm{M}-\mathrm{H}]^{-}\right)$ 519.1291; found 519.1289.

3.3.5. Oxidation of (4R-cis)-6-Hydroxymethyl-2,2-dimethyl-1,3-dioxane-4-acetic acid 1,1-dimethylethyl ester (1d):

Commercially available $1 \mathrm{~d}$ ( $26 \mathrm{mg}$, 1.0 equiv, $0.1 \mathrm{mmol}$ ) was oxidized according to the general procedure outlined above ( $5 \mathrm{~mol} \% \mathrm{TEMPO}, 5 \mathrm{~mol} \% \mathrm{TBAB}, 2.2$ equiv. $m \mathrm{CPBA}, 3 \mathrm{~h}$ reaction time), using benzene as the solvent and excluding the work-up sequence. The crude mixture was analyzed by NMR and was found to contain a 1:4.9 mixture of $\mathbf{2} \mathbf{d}$ and $\mathbf{3 d}$ [47]. Determination of $C$ was done using an overlapping spectrum of the pure starting material, the pure acid, the abovementioned 1:4.9 mixture, and TBAB.

2d: Partial ${ }^{1} \mathrm{H}-\mathrm{NMR}(400 \mathrm{MHz}, \mathrm{DMSO}-d 6): \delta 8.28(\mathrm{~s}, 1 \mathrm{H}), 6.14(\mathrm{dd}, J=9.6,3.0 \mathrm{~Hz}, 1 \mathrm{H}), 4.31(\mathrm{~m}, 1 \mathrm{H})$, $1.88(\mathrm{dt}, J=12.4,3.0 \mathrm{~Hz}, 1 \mathrm{H}) .{ }^{13} \mathrm{C}-\mathrm{NMR}(100 \mathrm{MHz}$, DMSO-d6): $\delta 169.2,161.2,100.0,89.2,79.9,65.2,64.1$, 41.6, 37.1, 34.4, 29.5, 20.7.

3d: ${ }^{1} \mathrm{H}-\mathrm{NMR}(400 \mathrm{MHz}$, DMSO-d6): $\delta 4.47(\mathrm{dd}, J=11.9,2.4 \mathrm{~Hz}, 1 \mathrm{H}), 4.26(\mathrm{~m}, 1 \mathrm{H}), 2.40(\mathrm{dd}, J=15.1$, $4.7 \mathrm{~Hz}, 1 \mathrm{H}), 2.23(\mathrm{dd}, J=15.2,8.1 \mathrm{~Hz}, 1 \mathrm{H}), 1.80(\mathrm{dt}, J=12.8,2.3 \mathrm{~Hz}, 1 \mathrm{H}), 1.40(\mathrm{~s}, 3 \mathrm{H}), 1.37(\mathrm{~s}, 9 \mathrm{H})$, 
$1.32(\mathrm{~m}, 1 \mathrm{H}), 1.28(\mathrm{~s}, 3 \mathrm{H}) .{ }^{13} \mathrm{C}-\mathrm{NMR}$ (100 MHz, DMSO-d6): $\delta 171.8,169.4,98.6,79.8,67.8,65.7,41.9,32.5$, $29.7,27.7,19.4$.

3.3.6. Oxidation of (5,5-Dimethyl-1,3-dioxan-2-yl)methanol (1e):

1e (146 mg, 1.0 equiv, $1.0 \mathrm{mmol}$ ) was oxidized according to the general procedure outlined above, scaled-up by ten ( $2 \mathrm{~mol} \%$ TEMPO, $2 \mathrm{~mol} \%$ TBAB, 3.3 equiv. $m \mathrm{CPBA}, 1 \mathrm{~h}$ reaction time), including the work-up sequence. Prior to extraction, the aqueous phase was saturated with solid $\mathrm{NaCl}$. The crude mixture of products was purified using column chromatography (silica gel, 1:9 to 3:7 EtOAc/hexane) to give the minor products $\mathbf{5 e - 1}$ (4 mg, impure with other by-products) and $\mathbf{5 e - 2}(3.5 \mathrm{mg})$, and the major product $4 \mathbf{e}(19 \mathrm{mg}, 0.14 \mathrm{mmol}, 14 \%)$ as colorless oils [48]. Due to their minute amounts, only ${ }^{1} \mathrm{H}-\mathrm{NMR}$ characterization is available for the minor products.

4e: TLC: $\mathrm{R}_{\mathrm{f}}=0.3\left(3: 7 \mathrm{EtOAc} /\right.$ hexane eluent). ${ }^{1} \mathrm{H}-\mathrm{NMR}\left(400 \mathrm{MHz}, \mathrm{CDCl}_{3}\right): \delta 8.10(\mathrm{~s}, 1 \mathrm{H}), 4.01(\mathrm{~s}, 2 \mathrm{H})$, $3.34(\mathrm{~s}, 2 \mathrm{H}), 2.11(\mathrm{br} \mathrm{s}, 1 \mathrm{H}), 0.93(\mathrm{~s}, 6 \mathrm{H}) .{ }^{13} \mathrm{C}-\mathrm{NMR}\left(100 \mathrm{MHz}, \mathrm{CDCl}_{3}\right): \delta 161.6,69.0,68.2,36.3,21.5$.

5e-1: ${ }^{1} \mathrm{H}-\mathrm{NMR}\left(400 \mathrm{MHz}, \mathrm{CDCl}_{3}\right): \delta 5.40(\mathrm{~s}, 1 \mathrm{H}), 4.66(\mathrm{t}, J=4.7 \mathrm{~Hz}, 1 \mathrm{H}), 3.76(\mathrm{~d}, J=11.0 \mathrm{~Hz}, 2 \mathrm{H})$, $3.71(\mathrm{~d}, J=4.7 \mathrm{~Hz}, 2 \mathrm{H}), 3.63(\mathrm{~d}, J=11.2 \mathrm{~Hz}, 2 \mathrm{H}), 3.44(\mathrm{~d}, J=10.6 \mathrm{~Hz}, 2 \mathrm{H}), 3.37(\mathrm{~d}, J=11.1 \mathrm{~Hz}, 2 \mathrm{H})$, $1.19(\mathrm{~s}, 3 \mathrm{H}), 0.98(\mathrm{~s}, 3 \mathrm{H}), 0.97(\mathrm{~s}, 3 \mathrm{H}), 0.73(\mathrm{~s}, 3 \mathrm{H})$.

5e-2: ${ }^{1} \mathrm{H}-\mathrm{NMR}\left(400 \mathrm{MHz}, \mathrm{CDCl}_{3}\right): \delta 8.10(\mathrm{~s}, 1 \mathrm{H}), 5.26(\mathrm{~s}, 1 \mathrm{H}), 4.03(\mathrm{~s}, 2 \mathrm{H}), 3.73(\mathrm{~d}, J=11.0 \mathrm{~Hz}, 2 \mathrm{H})$, $3.39(\mathrm{~s}, 2 \mathrm{H}), 3.38(\mathrm{~d}, J=10.5 \mathrm{~Hz}, 2 \mathrm{H}), 0.99(\mathrm{~s}, 9 \mathrm{H}), 0.95(\mathrm{~s}, 3 \mathrm{H})$.

3.3.7. Oxidation of ((3aR,9aS)-3a,4,9,9a-Tetrahydronaphtho[2,3-d][1,3]dioxol-2-yl)methanol (1f):

1f (21 mg, 1.0 equiv, $0.1 \mathrm{mmol}$ ) was oxidized according to the general procedure outlined above ( $2 \mathrm{~mol} \% \mathrm{TEMPO}, 2 \mathrm{~mol} \% \mathrm{TBAB}, 3.3$ equiv. $\mathrm{mCPBA}, 1 \mathrm{~h}$ reaction time), excluding the work-up sequence. The product $4 \mathrm{f}$ was characterized from the crude reaction mixture without purification.

${ }^{1} \mathrm{H}-\mathrm{NMR}(400 \mathrm{MHz}$, DMSO-d6): $\delta 8.28(\mathrm{~s}, 1 \mathrm{H}), 7.09(\mathrm{~m}, 4 \mathrm{H}), 5.17(\mathrm{t}, J=4.2 \mathrm{~Hz}, 1 \mathrm{H}), 4.07(\mathrm{ddd}$, $J=7.5,5.3,1.8 \mathrm{~Hz}, 1 \mathrm{H}), 2.94(\mathrm{~m}, 4 \mathrm{H}) .{ }^{13} \mathrm{C}-\mathrm{NMR}(100 \mathrm{MHz}$, DMSO-d6): $\delta$ 162.3, 133.9, 133.2, 128.7, 128.6, $125.9,125.8,75.2,72.4,34.3,31.9$.

3.3.8. Oxidation of (4,4,5,5-Tetramethyl-1,3-dioxolan-2-yl)methanol (1g):

$1 \mathrm{~g}$ (32 mg, 1.0 equiv, $0.2 \mathrm{mmol}$ ) was oxidized according to the general procedure outlined above ( $2 \mathrm{~mol} \%$ TEMPO, $2 \mathrm{~mol} \% \mathrm{TBAB}, 3.3$ equiv. $m \mathrm{CPBA}, 1 \mathrm{~h}$ reaction time), excluding the work-up sequence. The crude mixture was analyzed by NMR and was found to contain a 1:2.4 mixture of 4g [49] and 5 g [50,51].

4g: ${ }^{1} \mathrm{H}-\mathrm{NMR}\left(400 \mathrm{MHz}\right.$, DMSO-d6): $\delta 8.34(\mathrm{~s}, 1 \mathrm{H}), 1.37$ (s, 6H), $1.09(\mathrm{~s}, 6 \mathrm{H}) .{ }^{13} \mathrm{C}-\mathrm{NMR}(100 \mathrm{MHz}$, DMSO-d6): $\delta 162.6,85.9,72.9,25.0,22.1$. 22.0.

5g: ${ }^{1} \mathrm{H}-\mathrm{NMR}$ (400 MHz, DMSO-d6): $\delta 1.31$ (s, 12H). ${ }^{13} \mathrm{C}-\mathrm{NMR}$ (100 MHz, DMSO-d6): $\delta$ 153.3, 86.1,

\subsubsection{Oxidation of Methyl 3,5-di-O-(2,4-dichlorobenzyl)- $\alpha$-D-Ribofuranoside (1h):}

Commercially available $1 \mathrm{~h}$ ( $152 \mathrm{mg}, 0.3 \mathrm{mmol}, 1$ equiv) was oxidized according to the general procedure outlined above, scaled-up by three ( $5 \mathrm{~mol} \%$ TEMPO, $5 \mathrm{~mol} \% \mathrm{TBAB}, 2.2$ equiv. $m \mathrm{CPBA}$, $4 \mathrm{~h}$ reaction time), including the work-up sequence. The product was characterized from the crude reaction mixture without purification.

${ }^{1} \mathrm{H}-\mathrm{NMR}\left(400 \mathrm{MHz}, \mathrm{CDCl}_{3}\right): \delta 7.37(\mathrm{~m}, 2 \mathrm{H}), 7.30(\mathrm{~d}, J=8.2 \mathrm{~Hz}, 1 \mathrm{H}), 7.27(\mathrm{~d}, J=8.2 \mathrm{~Hz}, 1 \mathrm{H})$, $7.21(\mathrm{~d}, J=8.3 \mathrm{~Hz}, 1 \mathrm{H}), 7.21(\mathrm{~d}, J=8.3 \mathrm{~Hz}, 1 \mathrm{H}), 5.59(\mathrm{~d}, J=8.3 \mathrm{~Hz}, 1 \mathrm{H}), 5.00(\mathrm{~s}, 1 \mathrm{H}), 4.99(\mathrm{~d}, J=12.2 \mathrm{~Hz}$, $1 \mathrm{H}), 4.75(\mathrm{~d}, J=12.3 \mathrm{~Hz}, 1 \mathrm{H}), 4.63(\mathrm{~d}, J=13.0 \mathrm{~Hz}, 1 \mathrm{H}), 4.51(\mathrm{~d}, J=13.0 \mathrm{~Hz}, 1 \mathrm{H}), 4.09(\mathrm{dt}, J=8.3,3.0 \mathrm{~Hz}$, $1 \mathrm{H}), 3.82(\mathrm{~d}, J=3.0 \mathrm{~Hz}, 2 \mathrm{H}), 3.53(\mathrm{~s}, 3 \mathrm{H}) .{ }^{13} \mathrm{C}-\mathrm{NMR}\left(100 \mathrm{MHz}, \mathrm{CDCl}_{3}\right): \delta 163.4,135.2,134.5,134.3,133.9$, 133.6, 132.3, 131.1, 129.8, 129.6, 129.3, 127.4, 127.3, 100.0, 95.3, 70.3, 68.3, 68.2, 68.0, 56.9. 


\section{Conclusions}

In conclusion, we examined a number of additional classes of $\beta$-oxy-substituted primary alcohols in the domino two-step oxidation reaction that we disclosed recently. Furthermore, in a number of cases, formation of useful end-products was observed. Finally, the experiments helped to establish the order of migratory aptitudes of $\alpha$-oxy-substituted alkyls in the BV reaction and compared them to that of hydrogen $(\alpha$-hydroxyalkyl $>\alpha$-alkoxyalkyl $>\alpha, \alpha$-dialkoxyalkyl $>\alpha$-aryloxyalkyl $>$ hydrogen $>$ $\alpha$-alkoxyalkoxyalkyl $>\alpha$-acyloxyalkyl).

Supplementary Materials: The following are available online at http://www.mdpi.com/2073-4344/10/11/1275/s1, ${ }^{1} \mathrm{H}$ - and ${ }^{13} \mathrm{C}-\mathrm{NMR}$ spectra of the synthesized substrates and identified products, as well as indicative MS spectra of some of the compounds.

Author Contributions: Conceptualization and methodology, T.T. and M.P.; investigation and data curation, T.T.; writing-original draft preparation, T.T. and M.P.; writing-review and editing, M.P.; supervision, project administration and funding acquisition, M.P. All authors have read and agreed to the published version of the manuscript.

Funding: This research received no external funding.

Acknowledgments: The authors are grateful to Tel Aviv University for financial support.

Conflicts of Interest: The authors declare no conflict of interest.

\section{References and Notes}

1. Tojo, G.; Fernandez, M. Oxidation of Alcohols to Aldehydes and Ketones; Springer: New York, NY, USA, 2006.

2. Krow, G.R. The Baeyer-Villiger oxidation of ketones and aldehydes. Org. React. 1993, 43, 251-798.

3. ten Brink, G.J.; Arends, I.W.C.E.; Sheldon, R.A. The Baeyer-Villiger reaction: New developments toward greener procedures. Chem. Rev. 2004, 104, 4105-4123. [CrossRef]

4. Strukul, G. Transition metal catalysis in the Baeyer-Villiger oxidation of ketones. Angew. Chem. Int. Ed. 1998, 37, 1199-1209. [CrossRef]

5. Yaremenko, I.A.; Vil, V.A.; Demchuk, D.V.; Terent'ev, A.O. Rearrangements of organic peroxides and related processes. Beilstein. J. Org. Chem. 2016, 12, 1647-1748. [CrossRef] [PubMed]

6. For examples of oxidation of secondary alcohols to esters in two separate steps, see subsequent references 7-15, while for infrequent examples of one-pot oxidation of secondary alcohols to esters, see references 16-19.

7. Tsang, R.; Fraser-Reid, B. Pyranose alpha-enones provide ready access to functionalized trans-decalins via bis-annulated pyranosides obtained by intramolecular Diels-Alder reactions-a key intermediate for Forskolin. J. Org. Chem. 1992, 57, 1065-1067. [CrossRef]

8. Chida, N.; Tobe, T.; Ogawa, S. Regioselective Baeyer-Villiger reaction of polyhydroxycyclohexanone derivatives. Tetrahedron Lett. 1994, 35, 7249-7252. [CrossRef]

9. Mecerreyes, D.; Atthoff, B.; Boduch, K.A.; Trollsas, M.; Hedrick, J.L. Unimolecular combination of an atom transfer radical polymerization initiator and a lactone monomer as a route to new graft copolymers. Macromolecules 1999, 32, 5175-5182. [CrossRef]

10. Ishmuratov, G.Y.; Yakovleva, M.P.; Ganieva, V.A.; Gareeva, G.R.; Muslukhov, R.R.; Tolstikov, G.A. Synthesis of optically pure 3R-methylcyclopentan-1-one from L-(-)-menthol. Chem. Nat. Compd. 2005, 41, 549-551. [CrossRef]

11. Rainbolt, E.A.; Washington, K.E.; Biewer, M.C.; Stefan, M.C. Towards smart polymeric drug carriers: Self-assembling $\gamma$-substituted polycaprolactones with highly tunable thermoresponsive behavior. J. Mater. Chem. B 2013, 1, 6532-6537. [CrossRef]

12. Surnar, B.; Jayakannan, M. Stimuli-responsive poly(caprolactone) vesicles for dual drug delivery under the gastrointestinal tract. Biomacromolecules 2013, 14, 4377-4387. [CrossRef]

13. Ercole, F.; Rodda, A.E.; Meagher, L.; Forsythe, J.S.; Dove, A.P. Surface grafted poly( $\varepsilon$-caprolactone) prepared using organocatalysed ring-opening polymerisation followed by SI-ATRP. Polym. Chem. 2014, 5, 2809-2815. [CrossRef]

14. Yamauchi, S.; Nishimura, H.; Nishiwaki, H. Stereoselective syntheses of cryptocarya diacetate and all its stereoisomers in optically pure forms. Biosci. Biotechnol. Biochem. 2015, 79, 16-24. [CrossRef] 
15. Malhotra, M.; Surnar, B.; Jayakannan, M. Polymer topology driven enzymatic biodegradation in polycaprolactone block and random copolymer architectures for drug delivery to cancer cells. Macromolecules 2016, 49, 8098-8112. [CrossRef]

16. Cella, J.A.; McGrath, J.P.; Kelley, J.A.; El Soukkary, O.; Hilpert, L. Applications of the peracid-mediated oxidation of alcohols. J. Org. Chem. 1977, 42, 2077-2080. [CrossRef]

17. Chrobok, A. An efficient tandem oxidation of cyclohexanol to $\varepsilon$-caprolactone with peroxyacids and TEMPO catalyst in ionic liquids as solvents. Synlett 2011, 391-395. [CrossRef]

18. Weisser, F.; Stevens, H.; Klein, J.; van der Meer, M.; Hohloch, S.; Sarkar, B. Tailoring Ru(II) pyridine/triazole oxygenation catalysts and using photoreactivity to probe their electronic properties. Chem. Eur. J. 2015, 21, 8926-8938. [CrossRef]

19. Dijkmans, J.; Schutyser, W.; Dusselier, M.; Sels, B.F. Sn $\beta$-zeolite catalyzed oxido-reduction cascade chemistry with biomass-derived molecules. Chem. Commun. 2016, 52, 6712-6715. [CrossRef]

20. Figadere, B.; Franck, X. Carboxylic Acids: Synthesis from alcohols. In Science of Synthesis; Panek, J.S., Ed.; Georg Thieme Verlag: Stuttgart, Germany, 2006; Volume 20a, pp. 173-204.

21. For reactions of electron-rich benzaldehydes (Dakin reaction), see subsequent references 22-23, while for reactions of aldehydes with a nitrogen- or an oxygen-substituted $\alpha$-carbon, see references $24-31$.

22. Hocking, M.B.; Bhandari, K.; Shell, B.; Smyth, T.A. Steric and pH effects on the rate of Dakin oxidation of acylphenols. J. Org. Chem. 1982, 47, 4208-4215. [CrossRef]

23. Saikia, B.; Borah, P. A new avenue to the Dakin reaction in $\mathrm{H}_{2} \mathrm{O}_{2}-$ WERSA. RSC Adv. 2015, 5, 105583-105586. [CrossRef]

24. Alcaide, B.; Aly, M.F.; Sierra, M.A. Stereoselective synthesis of 3-substituted 4-(formyloxy)-2-azetidinones by the unusual Baeyer-Villiger reaction of beta-lactam aldehydes. Scope and synthetic applications. J. Org. Chem. 1996, 61, 8819-8825. [CrossRef]

25. Deboer, A.; Ellwanger, R.E. Baeyer-Villiger oxidation of $\Delta^{1(9)}$-Octalone-2 and $\Delta^{1(8)}$-Indanone-2. J. Org. Chem. 1974, 39, 77-83. [CrossRef]

26. Labadie, G.R.; Luna, L.E.; Gonzalez-Sierra, M.; Cravero, R.M. Synthesis of the tetracyclic bis(acetal) lactone portion of Saudin. Eur. J. Org. Chem. 2003, 3429-3434. [CrossRef]

27. Jeso, V.; Iqbal, S.; Hernandez, P.; Cameron, M.D.; Park, H.; LoGrasso, P.V.; Micalizio, G.C. Synthesis of benzoquinone Ansamycin-inspired macrocyclic lactams from Shikimic acid. Angew. Chem. Int. Ed. 2013, 52, 4800-4804. [CrossRef]

28. Nicolaou, K.C.; Frederick, M.O.; Burtoloso, A.C.B.; Denton, R.M.; Rivas, F.; Cole, K.P.; Aversa, R.J.; Gibe, R.; Umezawa, T.; Suzuki, T. Chemical synthesis of the GHIJKLMNO ring system of maitotoxin. J. Am. Chem. Soc. 2008, 130, 7466-7476. [CrossRef]

29. Chaubet, G.; Bourgeois, D.; Périgaud, C. Synthetic studies towards new nucleoside analogues: Preparation of $( \pm)-1^{\prime}, 4^{\prime}$-dimethyladenosine. Eur. J. Org. Chem. 2011, 319-326. [CrossRef]

30. Himmelbauer, M.; Farcet, J.B.; Gagnepain, J.; Mulzer, J. Palladium-catalyzed carbo-oxygenation: The Bielschowskysin case. Org. Lett. 2013, 15, 3098-3101. [CrossRef]

31. Urabe, F.; Nagashima, S.; Takahashi, K.; Ishihara, J.; Hatakeyama, S. Total synthesis of (-)-Cinatrin C1 based on an $\operatorname{In}(\mathrm{OTf})_{3}$-catalyzed Conia-ene reaction. J. Org. Chem. 2013, 78, 3847-3857. [CrossRef]

32. Targel, T.; Ramesh, P.; Portnoy, M. Domino two-step oxidation of $\beta$-alkoxy alcohols to hemiacetal esters: Linking a stoichiometric step to an organocatalytic step with a common organic oxidant. Eur. J. Org. Chem. 2018, 23, 3017-3021. [CrossRef]

33. For cases when such transformation was conducted in two successive, but separate, steps, see refs. 7,8.

34. For similar comparison of some of these migratory aptitudes in ketones, see ref. 8 .

35. The endocyclic character of the alkoxyalkyl should not be a factor in this comparison, since the related 3-hydroxytetrahydrofurane undergoes a rapid cascade reaction with the insertion of oxygen near this group.

36. Jimenez, F.; del Carmen Cruz, M.; Zuniga, C.; Martinez, M.A.; Chamorro, G.; Diza, F.; Tamariz, J. Aryloxyacetic esters structurally related to $\alpha$-Asarone as potential antifungal agents. Med. Chem. Res. 2010, 19, 33-57. [CrossRef]

37. $\mathbf{1 b}$ was prepared from a triol precursor by trans-acetalization with benzaldehyde dimethylacetal, see Materials and Methods. 
38. See, for instance: Xie, Y.; Zhang, J.; Tian, G.; Xu, M.; Hu, T.; Jiang, X.; Shen, J. A neighboring group participation strategy: Facile synthesis of 3,5-di-O-benzoyl-2-C-methyl-d-arabino- $\gamma$-lactone. Tetrahedron Lett. 2015, 56, 4345-4348. [CrossRef]

39. In order to drive the reaction to completion, excess of $m$ CPBA was applied.

40. Lehtinen, C.; Nevalainen, V.; Brunow, G. Experimental and computational studies on solvent effects in reactions of peracid-aldehyde adducts. Tetrahedron 2001, 57, 4741-4751. [CrossRef]

41. Ogata, Y.; Sawaki, Y. Kinetics of the Baeyer-Villiger reaction of benzaldehydes with perbenzoic acid in aquoorganic solvents. J. Org. Chem. 1969, 34, 3985-3991. [CrossRef]

42. Flogel, O.; Okala Amombo, M.G.; Reissig, H.U.; Zahn, G.; Brudgam, I.; Hartl, H. A stereoselective and short total synthesis of the polyhydroxylated $\gamma$-amino acid (-)-detoxinine, based on stereoselective preparation of dihydropyrrole derivatives from lithiated alkoxyallenes. Chem. Eur. J. 2003, 9, 1405-1415. [CrossRef]

43. Miyazawa, S.; Shinoda, M.; Kawahara, T.; Watanabe, N.; Harada, H.; Iida, D.; Terauchi, H.; Nagakawa, J.; Fujisaki, H.; Kubota, A.; et al. Preparation of Benzimidazole Derivatives as Gastric Acid Secretion Inhibitors. U.S. Patent Appl. Publ. US 2007/0010542 A1, 11 January 2007.

44. Huo, C.; Yang, H.; Cui, Q.C.; Dou, Q.P.; Chan, T.H. Proteasome inhibition in human breast cancer cells with high catechol-O-methyltransferase activity by green tea polyphenol EGCG analogs. Bioorg. Med. Chem. 2010, 18, 1252-1258. [CrossRef]

45. Lei, P.-s.; Ogawa, Y.; Kovac, P. New N-acylating reagent derived from 3-deoxy-L-glycero-tetronic acid. J. Carbohydr. Chem. 1996, 15, 485-500. [CrossRef]

46. Esmurziev, A.M.; Reimers, A.; Andreassen, T.; Simic, N.; Sundby, E.; Hoff, B.H. Benzoylated uronic acid building blocks and synthesis of N-uronate conjugates of Lamotrigine. Molecules 2012, 17, 820-835. [CrossRef]

47. For characterization of 3d, see: Edwards, J.T.; Merchant, R.R.; McClymont, K.S.; Knouse, K.W.; Qin, T.; Malins, L.R.; Vokits, B.; Shaw, S.A.; Bao, D.H.; Wei, F.-L.; et al. Decarboxylative alkenylation. Nature 2017, 545, 213-219.

48. For characterization of 4e, see: Filliatre, C.; Brigand, G.; Lalande, R. Peroxidation of oxygenated heterocyclic compounds. Bull. Soc. Chim. Fr. 1971, 170-176.

49. Goosen, A.; McCleland, C.W. Reaction of 1,3-dioxolans with iodine monochloride: The scope and mechanism of formation of 1,3-dioxolan-2-ylium dichloroiodates(I). J. Chem. Soc. Perkin Trans. I 1981, 977-983. [CrossRef]

50. Suzuki, M.; Sugai, T. Mechanistic studies on nitrosation-deaminocyclization of mono-carbamoylated vicinal amino alcohols and diols: A new preparative in situ formation of ethanediazo hydroxide for the ethylation of carboxylates under mild conditions. Bull. Chem. Soc. Jpn. 2004, 77, 1217-1227. [CrossRef]

51. Pihlaja, K.; Rossi, K. Conformational analysis. Part XLIII. ${ }^{13} \mathrm{C}$ Chemical shifts and coupling constants as proof of the nonplanarity of the 2-oxo-1,3-dioxalane ring. Acta Chem. Scand. B, 1977; 31, 899-902.

Publisher's Note: MDPI stays neutral with regard to jurisdictional claims in published maps and institutional affiliations.

(C) 2020 by the authors. Licensee MDPI, Basel, Switzerland. This article is an open access article distributed under the terms and conditions of the Creative Commons Attribution (CC BY) license (http://creativecommons.org/licenses/by/4.0/). 